\title{
Effect of changes in treatment practice on survival for cervical cancer: results from a population-based study in Manitoba, Canada
}

\author{
Yoon-Jung Kang ${ }^{1,2^{*}}$, Dianne L. O'Connell ${ }^{2}$, Robert Lotocki ${ }^{3,5}$, Erich V. Kliewer ${ }^{4,5}$, David E. Goldsbury²,
} Alain A. Demers ${ }^{4,5}$ and Karen Canfell ${ }^{1,2}$

\begin{abstract}
Background: Results from clinical trials in the 1990s led to changes in the recommended treatment for the standard therapy for stage IIB-IVA cervical cancer from radiotherapy alone to chemo-radiotherapy. We conducted the first population-based study in Canada to investigate temporal treatment patterns for cervical cancer and long-term survival in relation to these changes in the treatment guidelines.
\end{abstract}

Methods: Detailed information on stage and treatment for 1085 patients diagnosed with cervical cancer in 1984-2008 and identified from the population-based Manitoba Cancer Registry (MCR) in Canada was obtained from clinical chart review and the MCR. Factors associated with receiving guideline treatment were identified using logistic regression. All cause and cervical cancer specific survival were compared in patients who were and were not treated as recommended in the guidelines, using Cox proportional hazards models.

Results: The median follow-up time was 6.4 years (range: $0.05-26.5$ years). The proportion of women who received guideline treatment was $79 \%$ (95\% confidence interval [Cl]: 76-81\%). However, the likelihood of being treated according to the guidelines over time was modified by age $(p<0.0001)$ and tumour stage at diagnosis $(p=0.002)$. Women who were treated according to the guidelines after the change in recommended clinical practice (1999-2008) had a significantly lower risk of death from all causes and from cervical cancer. This was driven by lower mortality rates in cases with stage IIB-IVA tumours (all causes of death: hazard ratio $[\mathrm{HR}]=0.60,95 \% \mathrm{Cl}$ : 0.43-0.82, $p=0.002$; cervical cancer related death: $\mathrm{HR}=0.64,95 \% \mathrm{Cl}: 0.44-0.93, p=0.02$ ).

Conclusions: The management of cervical cancer patients in Manitoba, Canada was in good agreement with treatment guidelines although reasons for departure from the guideline recommendations could not be examined further due to lack of data. Treatment of stage IIB-IVA cervical cancers with recommended concurrent chemo-radiotherapy, which is now standard practice, was associated with substantially increased survival, although the effect of changes in clinical practice including maintenance of haemoglobin levels on improved survival cannot be ruled out as a contributing factor.

\section{Background}

Until the 1990s the standard therapy for International Federation of Gynecology and Obstetrics (FIGO) stage IIBIVA cervical cancer, or earlier stage disease with adverse pathological features, involved radiation alone. However, a rapid increase in concurrent use of chemo-radiotherapy has

\footnotetext{
* Correspondence: yoonjung.kang@nswcc.org.au

'Prince of Wales Clinical School, the University of New South Wales, Sydney, NSW, Australia

${ }^{2}$ Cancer Research Division, Cancer Council NSW, 153 Dowling Street,

Woolloomooloo, NSW, Australia

Full list of author information is available at the end of the article
}

occurred since the mid-1990s, after multi-centre randomised controlled trials (RCTs) [1-3] found cisplatinumbased concurrent chemo-radiotherapy prolonged survival in patients with advanced cervical cancer compared to radiotherapy alone. Subsequently, treatment guidelines in many jurisdictions [4-7] incorporated this new evidence. By contrast, the recommended treatments for early stage disease (FIGO Stage I-IIA), consisting of surgery with or without adjuvant radiotherapy, have not changed substantially over the last few decades. In Canada, guidelines for cervical cancer management have not been formulated at a national level, but the available provincial guidelines in 
Ontario [8,9] and British Columbia [10] do not substantially differ from the guidelines developed by the FIGO $[11,12]$ or available guidelines in other countries $[4,5,7]$. Therefore, "synthesised" guidelines, derived from available Canadian provincial and international guidelines, reflecting the available evidence can be readily formulated for Manitoba.

Studies from two Canadian centres in Ontario have investigated trends in the use of concurrent chemoradiotherapy and resulting improved survival outcomes in cervical cancer patients, without adjusting for tumour stage $[13,14]$. However, a population-based study investigating survival outcomes with long term follow up in women who were and were not managed in concordance with treatment guidelines has not previously been performed in the Canadian setting. Therefore, the aims of this study were to describe: 1) trends in treatment patterns in relation to changes in guideline recommendations; 2 ) the proportion of cervical cancer patients receiving treatment as recommended in the guidelines; 3 ) factors related to receiving treatment according to the guidelines; and 4) the impact of adhering to guidelines on the risk of death from all causes (i.e., any death) and from cervical cancer in the Canadian province of Manitoba.

\section{Methods}

\section{Study sample and data sources}

The population-based Manitoba Cancer Registry (MCR) was used to identify all incident cervical cancer cases diagnosed over the period 1984 to 2008 [15]. More detailed information on treatment was obtained by combining the MCR and a database derived from chart reviews (available only for the years 1984-1999); the registry and the charts are both maintained by CancerCare Manitoba.

Treatment procedures were coded using ICD-9-CM Volume 3 from 1984 to 2004 and the Canadian Classification of Health Interventions from 2005 to 2008: these two classification systems are comparable [16]. Morphologic data were coded using ICD-O-2 (1984-2000) and ICD-O-3 (2001-2008) that were comparable to each other. Cause of death was coded using ICD-9 until 1999 and ICD-10 thereafter. Although comparability between ICD-9 and ICD-10 on cause of death could not be examined for the current dataset, it was reported that there was a $2 \%$ increase in cervical cancer death when using ICD-10 compared to using ICD-9 [17].

Information on patients' performance status, comorbidities and recurrence were not recorded on either clinical chart or the MCR. Disease stage was defined according to the FIGO staging system (1984-1999) and equivalent American Joint Committee on Cancer (AJCC) staging system (2004-2008). For those who were diagnosed in 2000 to 2003, a stage based on the agreement between FIGO stage and clinical TNM category was used. This was based on the fact that the agreement between FIGO stage and clinical TNM staging, using the clinical chart review dataset that contains both staging information, was substantial (kappa $=0.74$, weighted kappa $=0.83)$ [18]. The agreement between the two staging systems was relatively lower for patients with stage IB2-IIA disease (68\%), but the proportions under-staged or over-staged were similar (15\% vs $18 \%$, respectively).

During 1984 to 2008, a total of 1413 incident cases of cervical cancer were identified from the MCR. For the overlapping period 1984 to 1999 , the reliability of the two data sets was examined by comparing seven indicators including the number of cases diagnosed in each year, date of diagnosis, age at diagnosis, treatment procedures and related dates, histology and cause of death. During the period, the total number of patients identified from any of the two data sets was 1043. Of these, $845(81 \%)$ were found in both data sets, and the remaining number of patients included 87 non-residents in the clinical chart review and 111 residents in the clinical cancer registry. For the 845 patients identified in both data sets, there was full agreement for six out of the seven indicators. The only exception was cause of death. For the time period 1984 to 1999, the MCR was used to determine the vital status if the information in the MCR and the chart review was inconsistent (12 out of 845 patients). For 328 patients there was either no tumour stage information and/or they received no treatment: 264 patients had no tumour stage information; 53 patients had no treatment records and it was not possible to identify whether they did or did not receive any treatment; for 11 patients neither FIGO stage nor treatment records were available. The final study sample consisted of 1085 (77\%) cervical cancer cases. There were no differences in demographic and clinical characteristics for those included and not included in the analysis (results not shown).

\section{Treatment recommendations in the guidelines}

As there are no published national guidelines for cervical cancer treatment in Canada, synthesised guidelines were derived from available provincial (published 2002 onwards) and international (FIGO) consensus and evidence-based treatment guidelines. Although changes occurred over time, there were no substantial differences identified between the provincial and the FIGO guidelines. For the purpose of the analysis, the FIGO guidelines were used as reference to evaluate the clinical practice in Manitoba for the years 1984 to 1998 [12, 19]. For 1999 to 2008, the synthesised evidence-based guidelines were used (Table 1) $[4-7,11]$. It was not possible to determine if the course of the treatment was completed or if the treatment schedule/doses were modified due to intolerance 
or choices by physicians and/or patients, due to data availability. Similarly, the exact timing and mechanisms for the guideline implementation in the local setting were not available from the administrative data.

\section{Statistical analysis}

The overall trend in the initial treatment during 1984 and 2008 was described using the 3 -year average (Fig. 1). Treatment patterns for cervical cancer patients by diagnosis period (1984-1998, 1999-2008) stratified by tumour stage (IA, IB-IIA, IIB-IVA and IVB) were cross-tabulated. Bivariable analyses were conducted to examine differences in demographic and tumour characteristics of women who did and did not receive treatment recommended in the guidelines.

A binomial logistic regression model was fitted to identify the factors associated with receiving guideline treatment (i.e., treatment according to the guidelines). Factors examined included tumour stage; age at diagnosis (0-45 years, 46-65 years, >65 years); diagnosis period (1984-1998, 1999-2008); histology (squamous cell carcinoma or adenosquamous carcinoma, adenocarcinoma or other histology); and area of residence (urban [Winnipeg and Brandon], rural).

A Cox proportional hazards regression model was used to examine the association between receiving the treatments as recommended and the risk of death from all causes and from cervical cancer. Time to death was calculated from the date of diagnosis to the date of death or censored at 30 June 2010. Potential confounders included diagnosis period, age, histology, area of residence and tumour stage. Stratified analysis by tumour stage was also performed. Data were analysed using SAS 9.2 (SAS Institute Inc., Cary, NC, USA).

\section{Ethics approval}

The study obtained human research ethics approval from the University of Manitoba Health Research Ethics Boards, the University of Sydney Human Research Ethics Committee and Cancer Council NSW Human Research Ethics Committee. As this study used de-identified data, all Human Research Ethics Committees waived the need for consent to participate in this study.

\section{Results}

\section{Baseline characteristics}

The median age at diagnosis of invasive cervical cancer was 50 years (range: $16-89$ ). The majority (74 \%) of patients were diagnosed with either stage IB to IIA or IIB to IVA disease. The proportion of women over 65 years of age was greater in those diagnosed with stage IIB-IVB disease than those with stage IA-IIA disease (32\% and $14 \%$, respectively). The majority of patients lived in urban areas at the time of diagnosis (65\%). Squamous cell carcinoma (including adenosquamous carcinoma) was the most common histology type (80 \%) (Table 2). Surgery alone was the most frequently used treatment for patients with IA and IB-IIA stage disease (93\% and $44 \%$, respectively), whereas radiotherapy alone was the most frequently used therapy for patients with IIB-IVA and IVB stage disease (60\% and $73 \%$, respectively) (Table 3).

Table 1 Synthesised guidelines for treatment of cervical cancer cases

\begin{tabular}{|c|c|c|}
\hline \multirow[b]{2}{*}{ FIGO stage } & \multicolumn{2}{|l|}{ Recommended treatment } \\
\hline & Consensus guidelines $^{a}[12,19]$ (applicable to 1998) & $\begin{array}{l}\text { Synthesised evidence-based guidelines [4-7, 11] (applicable from } \\
1999 \text { onwards) }\end{array}$ \\
\hline $\mid \mathrm{A} 1$ & $\begin{array}{l}\text { Total hysterectomy, conisation, radical hysterectomy }{ }^{\mathrm{b}} \text {, } \\
\text { radiotherapy }\end{array}$ & Total hysterectomy, conisation, radiotherapy \\
\hline IA2 & Radical hysterectomy, total hysterectomy, radiotherapyc & $\begin{array}{l}\text { Radical hysterectomy, total hysterectomy }{ }^{d} \text {, trachelectomy, } \\
\text { radiotherapy }{ }^{c}\end{array}$ \\
\hline \multicolumn{3}{|l|}{$\mid B-\| A$} \\
\hline \multirow[t]{2}{*}{$\leq 4 \mathrm{~cm}$} & \multirow[t]{2}{*}{ Radical hysterectomy, radiotherapy } & Radical hysterectomy, radiotherapy, \\
\hline & & Radical hysterectomy + adjuvant radiotherapy (chemo-radiotherapy) \\
\hline \multirow[t]{2}{*}{$>4 \mathrm{~cm}$} & \multirow{2}{*}{$\begin{array}{l}\text { Radical hysterectomy, radical hysterectomy + adjuvant } \\
\text { radiotherapy }\end{array}$} & Radical hysterectomy, chemo-radiotherapy, \\
\hline & & Radical hysterectomy + adjuvant radiotherapy(chemo-radiotherapy) \\
\hline IIB-IVA & Chemo-radiotherapy, radiotherapy & Chemo-radiotherapy \\
\hline IVB & Radiotherapy (curative/palliative), chemotherapy & Radiotherapy (curative/palliative), chemotherapy, chemo-radiotherapy \\
\hline
\end{tabular}




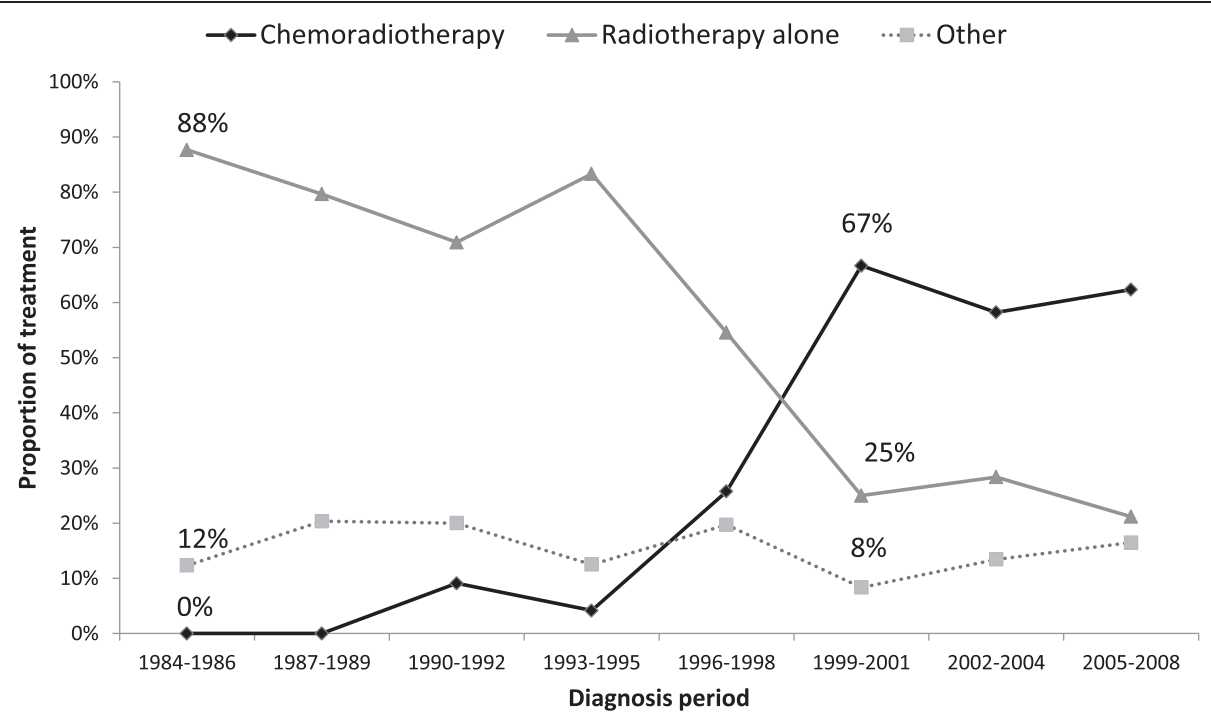

Fig. 1 Trends in the initial treatment for cervical cancer patients diagnosed with stage IB2-IVA tumours $(n=513)$. Other treatment includes surgery alone, chemotherapy alone, pre-operative radiation followed by surgery, surgery with adjuvant chemotherapy, surgery with adjuvant radiotherapy, palliative radiation and no treatment

Table 2 Characteristics of cervical cancer cases diagnosed in 1984-2008 by adherence to treatment guidelines $\left(n=1085^{\mathrm{a}}\right)$

\begin{tabular}{|c|c|c|c|c|}
\hline \multirow[b]{3}{*}{ Characteristics } & \multicolumn{3}{|c|}{ Treated according to treatment guidelines } & \multirow[b]{3}{*}{$p$-value* } \\
\hline & Total & $\begin{array}{l}\text { Yes } \\
(n=852)\end{array}$ & $\begin{array}{l}\text { No } \\
(n=233)\end{array}$ & \\
\hline & No. (Column \%) & No. (Row \%) & No. (Row \%) & \\
\hline Tumour stage & & & & 0.005 \\
\hline IA & $230(21)$ & $175(76)$ & $55(24)$ & \\
\hline $\mid \mathrm{B}-\| \mathrm{A}$ & $400(37)$ & $301(75)$ & $99(25)$ & \\
\hline IIB-IVA & $435(37)$ & $326(75)$ & $109(25)$ & \\
\hline IVB & $52(5)$ & $50(96)$ & $2(4)$ & \\
\hline Age at diagnosis & & & & 0.22 \\
\hline $0-45$ & $518(48)$ & $398(77)$ & $120(23)$ & \\
\hline $46-65$ & $333(31)$ & $261(78)$ & $72(22)$ & \\
\hline$>65$ & $234(22)$ & $193(82)$ & $41(18)$ & \\
\hline Histology & & & & 0.04 \\
\hline SCC & $866(80)$ & $691(80)$ & $175(20)$ & \\
\hline Others & $219(20)$ & $161(74)$ & $58(26)$ & \\
\hline $\begin{array}{l}\text { Area of } \\
\text { residence }\end{array}$ & & & & 0.90 \\
\hline Urban & $707(65)$ & $554(78)$ & $153(22)$ & \\
\hline Rural & $378(35)$ & $298(79)$ & $80(21)$ & \\
\hline Diagnosis period & & & & $<.0001$ \\
\hline 1984-1998 & $718(66)$ & $594(83)$ & $124(17)$ & \\
\hline 1999-2008 & 367 (34) & $258(70)$ & $109(30)$ & \\
\hline
\end{tabular}

SCC squamous cell carcinoma/adenosquamous carcinoma *For chi-square test of association

${ }^{a}$ After excluding cases with either missing stage data ( $14 \%$, mostly diagnosed in 2000-2003), cause of death (4\%) or treatment records (5\%)

\section{Overall trends in the initial treatment}

During the period 1984 to 2008, use of chemo-radiotherapy increased with a concomitant decrease in the use of radiotherapy alone, especially for patients with tumour staged IB2-IVA (Fig. 1). Until 1995, the predominant initial treatment (i.e., treatment given within the first year after diagnosis) for stage IB2-IVA tumours was radiotherapy alone. The use of combined chemo-radiotherapy started to increase steadily from $4 \%$ in 1993-1995 and became the predominant treatment from 1999 onward (67 \% of women).

\section{Guideline treatment}

Most women with invasive cervical cancer in Manitoba received guideline treatment (79 \%, $95 \%$ CI: 76-81 \%) over the study period. The overall proportion of women receiving guideline treatment was higher in 1984-1998 (83 \%, 95 \% CI: 80-85 \%) than in 1999-2008 (70\%, $95 \%$ CI: $65-75 \%)$. The proportion of women receiving guideline treatment by tumour stage in the later period did not substantially differ from that in the earlier period, with the exception of stage IIB-IVA (92 \% vs $64 \%$ ) where a substantial proportion of women received radiotherapy alone instead of chemo-radiotherapy in the later period (Table 3).

The bivariable analysis showed an association between receipt of treatment according to the guidelines and tumour stage $(p=0.005)$, tumour histology $(p=0.04)$ and time period $(p<0.0001)$ (Table 2$)$. The effect of time period on the odds of receiving guideline treatment was modified by both stage $(p=0.002)$ and age at diagnosis $(p<0.0001)$ (Table 4). Compared with those who were diagnosed in 1984-1998, patients diagnosed with stage IA or IB-IIA tumours in 1999-2008 at over 65 years of 
Table 3 Treatment administered to cervical cancer cases by tumour stage and time period (1984-1998 and 1999-2008) (n=1085)

\begin{tabular}{|c|c|c|c|c|c|c|c|c|c|c|c|c|c|c|c|c|}
\hline \multirow[b]{4}{*}{ Treatment } & \multicolumn{16}{|c|}{ Women treated for cervical cancer according to the guidelines by tumour stage and time period } \\
\hline & \multicolumn{4}{|c|}{ Stage IA $(n=229)$} & \multicolumn{4}{|c|}{ Stage IB-\| $A^{b}(n=398)$} & \multicolumn{4}{|c|}{ Stage IIB-IVA $(n=406)$} & \multicolumn{4}{|c|}{ Stage IVB $(n=52)$} \\
\hline & \multicolumn{2}{|l|}{$1984-1998$} & \multicolumn{2}{|c|}{ 1999-2008 } & \multicolumn{2}{|c|}{ 1984-1998 } & \multicolumn{2}{|c|}{ 1999-2008 } & \multicolumn{2}{|c|}{ 1984-1998 } & \multicolumn{2}{|c|}{ 1999-2008 } & \multicolumn{2}{|c|}{ 1984-1998 } & \multicolumn{2}{|c|}{ 1999-2008 } \\
\hline & No./Total & $\%$ & No./Total & $\%$ & No./Total & $\%$ & No./Total & $\%$ & No./Total & $\%$ & No./Total & $\%$ & No./Total & $\%$ & No./Total & $\%$ \\
\hline Surgery alone & $121 / 152$ & 80 & $46 / 62$ & 74 & $95 / 138$ & 69 & $32 / 36$ & 89 & $0 / 3$ & 0 & $0 / 2$ & 0 & $0 / 0$ & - & $0 / 0$ & - \\
\hline Surgery + adjuvant radiotherapy & $0 / 7$ & 0 & $0 / 0$ & - & $32 / 44$ & 73 & $8 / 14$ & 57 & $0 / 2$ & 0 & $0 / 9$ & 0 & $0 / 0$ & - & $0 / 1$ & 0 \\
\hline Preoperative radiotherapy + surgery & $0 / 0$ & - & $0 / 0$ & - & $5 / 7$ & 71 & $0 / 2$ & 0 & $0 / 14$ & 0 & $0 / 0$ & - & $0 / 0$ & - & $0 / 0$ & - \\
\hline Surgery + adjuvant chemotherapy & $0 / 0$ & - & $0 / 0$ & - & $0 / 2$ & 0 & $0 / 0$ & - & $0 / 0$ & - & $0 / 0$ & - & $0 / 0$ & - & $0 / 1$ & 0 \\
\hline Radiotherapy alone & $7 / 7$ & 100 & $1 / 1$ & 100 & $99 / 99$ & 100 & $8 / 9$ & 89 & 196/196 & 100 & $0 / 49$ & 0 & $17 / 17$ & 100 & $21 / 21$ & 100 \\
\hline Chemo-radiotherapy & $0 / 0$ & - & $0 / 0$ & - & $0 / 6$ & 0 & $22 / 38$ & 58 & $22 / 22$ & 100 & 108/108 & 100 & $0 / 0$ & - & $10 / 10$ & 100 \\
\hline Chemotherapy alone & $0 / 0$ & - & $0 / 0$ & - & $0 / 2$ & 0 & $0 / 1$ & 0 & $0 / 0$ & - & $0 / 1$ & 0 & $0 / 0$ & - & $2 / 2$ & 100 \\
\hline Total & $128 / 166$ & 77 & $47 / 63$ & 75 & $231 / 298$ & 78 & $70 / 100$ & 70 & $218 / 237$ & 92 & 108/169 & 64 & 17/17 & 100 & $33 / 35$ & 94 \\
\hline
\end{tabular}

$\%(95 \% \mathrm{Cl})$ of women treated according to the guidelines
By tumour stage and time period
$77 \%(70-83 \%)$
$75 \%(62-85 \%)$
$78 \%(72-82 \%) \quad 70 \%(60-79 \%)$
$92 \%(88-95 \%)$
$64 \%(56-71 \%)$
$100 \%(80-100 \%) \quad 94 \%(81-99 \%)$
By tumour stage in 1984-2008
$76 \%(70-82 \%)$ in 1984-2008 $\quad 76 \%(71-80 \%)$ in 1984-2008
$80 \%(76-84 \%)$ in 1984-2008
$96 \%(87-100 \%)$ in $1984-2008$

By time period for all tumour stages

Overall

$79 \%(76-81 \%)$ in $1984-2008$

No. number of women treated according to the guidelines

${ }^{a}$ Most patients who did not receive guideline treatment were treated with different surgery types (for example, LEEP with or without hysterectomy or total hysterectomy where radical hysterectomy was indicated or

vice versa)
${ }^{b}$ Most patients who were not treated according to the guidelines were treated with total hysterectomy with or without adjuvant radiotherapy. Patients with bulky lesion and treated with chemo-radiotherapy were regarded as not receiving guideline treatment

regarded as not receiving guideline treatment
CPatients diagnosed with advanced stage disease who received radiotherapy alone due to co-morbidities or poor performance status were regarded as not receiving guideline treatment 
Table 4 Factors associated with the probability of being treated according to treatment guidelines for cervical cancer cases diagnosed in 1984-2008 ( $n=1033)$

\begin{tabular}{|c|c|c|c|c|c|}
\hline \multicolumn{3}{|l|}{ 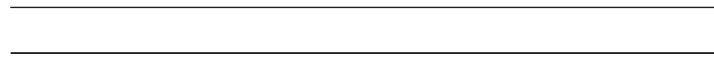 } & Total no. cases & Adjusted OR $(95 \% \mathrm{Cl})^{a}$ & $p$-value \\
\hline \multicolumn{5}{|l|}{ Histology } & 0.09 \\
\hline \multicolumn{3}{|l|}{ SCC } & 854 & 1.00 & \\
\hline \multicolumn{3}{|l|}{ Others } & 211 & $0.72(0.50-1.05)$ & \\
\hline \multicolumn{3}{|c|}{ Area of residence } & & & 0.77 \\
\hline \multicolumn{3}{|l|}{ Urban } & 691 & 1.00 & \\
\hline \multicolumn{3}{|l|}{ Rural } & 374 & $0.95(0.69-1.32)$ & \\
\hline \multicolumn{6}{|c|}{ Tumour stage ${ }^{\mathrm{b}}$ by time period and age at diagnosis } \\
\hline \multirow[t]{6}{*}{$\mathrm{IA}$} & $0-45$ years* $^{*}$ & $1984-1998^{* *}$ & 119 & 1.00 & \\
\hline & & 1999-2008 & 50 & $0.99(0.49-1.99)$ & \\
\hline & $46-65$ years & 1984-1998 & 35 & 1.00 & \\
\hline & & 1999-2008 & 11 & $0.97(0.40-2.37)$ & \\
\hline & $>65$ years & 1984-1998 & 13 & 1.00 & \\
\hline & & 1999-2008 & 2 & $0.11(0.04-0.33)$ & \\
\hline \multirow[t]{6}{*}{$|\mathrm{B}-| \mid \mathrm{A}$} & $0-45$ years & 1984-1998 & 172 & 1.00 & \\
\hline & & 1999-2008 & 50 & $1.04(0.57-1.90)$ & \\
\hline & $46-65$ years & 1984-1998 & 72 & 1.00 & \\
\hline & & 1999-2008 & 31 & $1.02(0.50-2.08)$ & \\
\hline & $>65$ years & 1984-1998 & 56 & 1.00 & \\
\hline & & 1999-2008 & 19 & $0.11(0.04-0.29)$ & \\
\hline \multirow[t]{6}{*}{ IIB-IVA } & $0-45$ years & 1984-1998 & 65 & 1.00 & \\
\hline & & 1999-2008 & 57 & $0.28(0.13-0.57)$ & \\
\hline & $46-65$ years & 1984-1998 & 96 & 1.00 & \\
\hline & & 1999-2008 & 72 & $0.27(0.14-0.54)$ & \\
\hline & $>65$ years & 1984-1998 & 104 & 1.00 & \\
\hline & & 1999-2008 & 41 & $0.03(0.01-0.08)$ & \\
\hline
\end{tabular}

$O R$ odds ratio, SCC squamous cell carcinoma/adenosquamous carcinoma

*Interaction between time period and age $(p<0.0001)$

**Interaction between time period and tumour stage $(p=0.002)$

${ }^{\mathrm{a}} \mathrm{OR}$ was adjusted for all variables shown in this table

${ }^{b}$ Patients with tumour stage IVB were not included in the analysis due to insufficient number

age were significantly less likely to receive treatment according to the guidelines $(\mathrm{OR}=0.11$ in both stage groups). For patients diagnosed with stage IIB-IVA disease in the later period, women in all age groups were less likely to receive treatment according to the guidelines compared with those who were diagnosed in the earlier period.

\section{Effect of guideline treatment on survival}

The median follow-up time after diagnosis was 6.4 years (range: 0.05-26.50 years). The overall number of deaths due to cervical cancer and all causes was 312 and 473, respectively. Among patients diagnosed with stage IA disease and who were not treated according to the guidelines, there were no deaths from cervical cancer. Therefore, cervical cancer death probabilities were determined for those diagnosed with stage IB-IVB tumours only.

\section{All-cause mortality}

The risk of dying from any cause following a cervical cancer diagnosis increased with the stage of the disease and with increasing age at diagnosis $(p<0.0001)$ (Table 5). The effect of being treated according to the guidelines on all-cause mortality differed over the diagnosis period $\left(P_{\text {interaction }}=0.0001\right)$. Patients diagnosed in 1984-1998 and who were treated according to the guidelines had a similar risk of dying to those who did not $(\mathrm{HR}=1.22,95 \% \mathrm{CI}: 0.85-1.75)$. By contrast, women diagnosed 1999-2008 and who were treated according to the guidelines experienced a $56 \%$ decreased risk of death from all causes (HR $=0.44,95 \% \mathrm{CI}$ : 0.31-0.64). Histology $(p=0.47)$ and area of residence $(p=0.06)$ were not significantly associated with the risk of dying from all causes. The tumour stage stratified analysis (Table 6) showed that independent effects of being treated according to the guidelines 
Table 5 Association between receipt of guideline treatment and the probability of dying for cervical cancer patients (all stage groups) who were diagnosed 1984-2008 $(n=1085)$

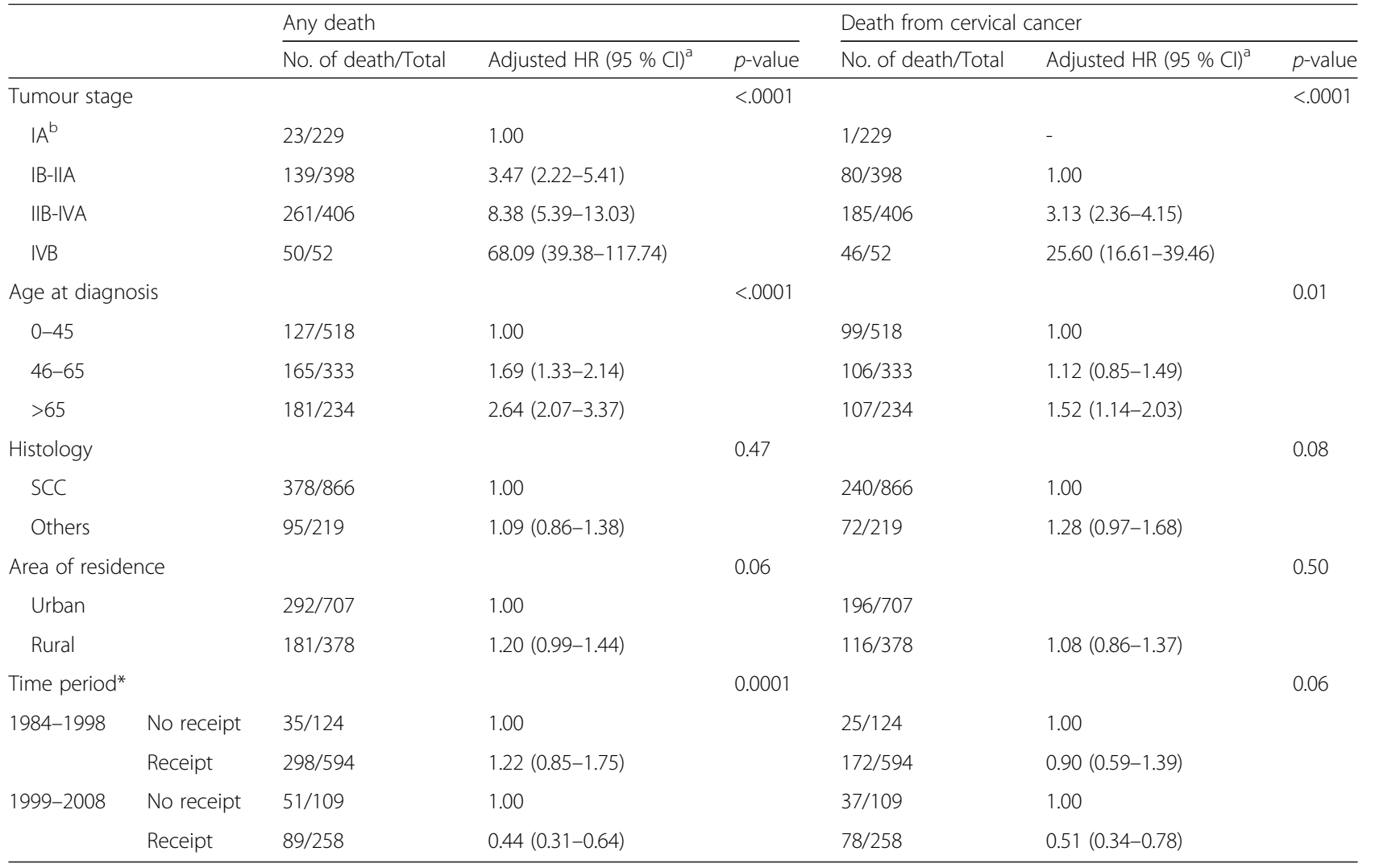

HR hazard ratio, SCC squamous cell carcinoma/adenosquamous carcinoma, No receipt did not receive guideline treatment, Receipt Received guideline treatment ${ }^{*} p$-value for interaction between time period and treatment according to the guidelines

${ }^{\mathrm{a}} \mathrm{HR}$ was adjusted for all variables shown in this table

${ }^{b} \mathrm{HR}$ for patients with tumour stage IA was not considered since none died from cervical cancer in the group who were not treated according to the guidelines

as well as being diagnosed in the later period, were only observed for stage IIB-IVA disease, i.e., women who received concurrent chemo-radiotherapy had a significantly decreased risk of death from all causes $(\mathrm{HR}=0.60,95 \% \mathrm{CI}$ : $0.43-0.82, p=0.002$ ).

\section{Cause-specific death}

The risk of death from cervical cancer increased with tumour stage at diagnosis $(p<0.0001)$ and in women diagnosed over 65 years of age $(p=0.01)$ (Table 5). The effect of the adherence to the guidelines was found to be weakly modified by diagnosis period $\left(P_{\text {interaction }}=0.06\right)$. For those diagnosed in 1984-1998, adherence to treatment guidelines did not impact the probability of dying from cervical cancer $(H R=0.90$, 95 \% CI: 0.59-1.39) (Table 5). By contrast, women diagnosed in 1999-2008 and who were treated according to the guidelines had a reduced risk of dying from cervical cancer $(\mathrm{HR}=0.51,95 \% \mathrm{CI}$ : 0.34-0.78). The tumour stage stratified analysis showed independent effects of receiving treatment according to the guidelines, and time period in those with stage IIB-IVA disease (Table 6). Women with stage IIB-IVA disease who received concurrent chemo-radiotherapy, had a significantly decreased risk of death from cervical cancer $(\mathrm{HR}=0.64,95 \% \mathrm{CI}: 0.44-0.93, p=0.02)$.

\section{Discussion}

Brief summary of the main results

A shift from radiotherapy alone to concurrent chemoradiotherapy as the predominant treatment for IB2-IVA stage cervical cancer cases was observed since 1999 in Manitoba, Canada, which was concordant with the changes in the published treatment guidelines $[4-7,11]$. The likelihood of receiving the guideline treatment, as well as the effect of guideline treatment on survival, varied by diagnosis period and tumour stage. Women diagnosed with stage IIB-IVA disease since 1999 were less likely to receive guideline treatment compared with those diagnosed earlier. This finding may to be due, in part, to older women not receiving concurrent chemoradiotherapy because of the presence of comorbidities, poorer health or choice. The significant reduction in the risk of death from both all causes (56 \%) and from 
Table 6 Effects of being treated according to the guidelines and time period on the probability of dying from all causes and cervical cancer by tumour stage $(n=1085)$

\begin{tabular}{|c|c|c|c|c|c|c|}
\hline & \multicolumn{3}{|l|}{ Any death } & \multicolumn{3}{|c|}{ Death from cervical cancer } \\
\hline & No. of death/Total & Adjusted HR $(95 \% \mathrm{Cl})^{\mathrm{a}}$ & $p$-value & No. of death/Total & Adjusted HR $(95 \% \mathrm{Cl})^{\mathrm{a}}$ & $p$-value \\
\hline \multicolumn{7}{|l|}{ Stage $\mid A^{b}$} \\
\hline \multicolumn{7}{|c|}{ Treated according to guidelines } \\
\hline No & $1 / 54$ & 1.00 & 0.08 & $0 / 54$ & - & \\
\hline Yes & $22 / 175$ & $5.98(0.80-44.83)$ & & $1 / 175$ & & \\
\hline \multicolumn{7}{|l|}{ Time period } \\
\hline 1984-1998 & $22 / 166$ & 1.00 & 0.41 & $1 / 166$ & - & \\
\hline 1999-2008 & $1 / 63$ & $0.42(0.05-3.28)$ & & $0 / 63$ & & \\
\hline \multicolumn{7}{|l|}{ Stage IB-\|IA } \\
\hline \multicolumn{7}{|c|}{ Treated according to guidelines } \\
\hline No & 29/97 & 1.00 & 0.84 & 19/97 & 1.00 & 0.43 \\
\hline Yes & $110 / 301$ & $0.96(0.63-1.47)$ & & $61 / 301$ & $0.81(0.48-1.38)$ & \\
\hline \multicolumn{7}{|l|}{ Time period } \\
\hline 1984-1998 & $116 / 298$ & 1.00 & 0.50 & $64 / 298$ & 1.00 & 0.45 \\
\hline 1999-2008 & $23 / 100$ & $0.85(0.53-1.37)$ & & $16 / 100$ & $0.80(0.45-1.43)$ & \\
\hline \multicolumn{7}{|l|}{ Stage IIB-IVA } \\
\hline \multicolumn{7}{|c|}{ Treated according to guidelines } \\
\hline No & $54 / 80$ & 1.00 & 0.002 & $41 / 80$ & 1.00 & 0.02 \\
\hline Yes & $207 / 326$ & $0.60(0.43-0.82)$ & & $144 / 326$ & $0.64(0.44-0.93)$ & \\
\hline \multicolumn{7}{|l|}{ Time period } \\
\hline 1984-1998 & $178 / 237$ & 1.00 & 0.004 & $115 / 237$ & 1.00 & 0.04 \\
\hline 1999-2008 & $83 / 169$ & $0.65(0.48-0.87)$ & & $70 / 169$ & $0.71(0.51-0.98)$ & \\
\hline \multicolumn{7}{|l|}{ Stage IVB } \\
\hline \multicolumn{7}{|c|}{ Treated according to guidelines } \\
\hline No & $2 / 2$ & 1.00 & 0.63 & $2 / 2$ & 1.00 & 0.55 \\
\hline Yes & $48 / 50$ & $0.68(0.14-3.30)$ & & $44 / 50$ & $0.62(0.13-3.02)$ & \\
\hline \multicolumn{7}{|l|}{ Time period } \\
\hline 1984-1998 & $17 / 17$ & 1.00 & 0.03 & $17 / 17$ & 1.00 & 0.02 \\
\hline 1999-2008 & $33 / 35$ & $0.43(0.20-0.93)$ & & $29 / 35$ & $0.38(0.17-0.84)$ & \\
\hline
\end{tabular}

${ }^{a} \mathrm{HR}$ was adjusted for age at diagnosis, histology, area of residence, time period and treatment according to the guidelines

${ }^{\mathrm{b}}$ The HR for patients diagnosed with tumours stage IA patients was not calculated because there were no deaths from cervical cancer

cervical cancer (49 \%) was observed only in those who were diagnosed since 1999 and received guideline treatment, and this appeared to be largely driven by the use of concurrent chemo-radiotherapy in stage group IIB-IVA.

\section{Explanation for the findings}

Previous studies reported a rapid increase in the use of concurrent chemo-radiotherapy following the USA National Cancer Institute's clinical announcement in 1999, which strongly encouraged cisplatinum-based concurrent chemo-radiotherapy for advanced stage cervical cancer [13, 14]. However, the current study observed that the change in management practice was already occurring while the related RCTs were being conducted. This implies that clinicians could have been aware of the trials from scientific meetings before the results were published in peer reviewed journals, and were ready to adopt the new evidence into the management of advanced cervical cancer. A similar phenomenon has been reported in the treatment of other cancer types, for example in the use of taxanes for primary breast cancer. The use of Paclitaxel substantially increased in the year following the presentation of study findings at the American Society of Clinical Oncology (ASCO) meeting in 1998, but the study was not published in a peer reviewed journal until five years later [20].

In agreement with previous reports, this study also found that older women were less likely to receive concurrent 
chemo-radiotherapy $[13,21]$. Chemo-radiotherapy is associated with acute haematological, renal and gastrointestinal toxicity [22]. Therefore, patients with poor performance status and co-morbid conditions received radiotherapy alone [13]. The main reason for patients not receiving chemo-radiotherapy in Manitoba was poor renal function at diagnosis (Personal communication, Dr Robert Lotocki, CancerCare Manitoba, Canada), although detailed information was not available to adjust for treatment uptake and survival in the current analysis.

The improved survival found in this study could potentially have resulted from a range of factors, including the use of concurrent chemo-radiotherapy as well as instituting a policy of maintaining a patient's haemoglobin to greater than $120 \mathrm{~g} / \mathrm{L}$ using blood transfusion while on treatment [23]. Studies from two Canadian centres in Ontario have investigated trends in the use of concurrent chemo-radiotherapy and the resulting improved survival outcomes, although they have not included stage-specific analyses [13, 14]. An American study reported that patients who did not receive guideline treatment experienced similar survival to those who did in 1988-1994 [24]. In the current study, women who were treated according to the guidelines in 1984 and 1998 experienced the same risk of death as those who were not treated according to the guidelines. By contrast, a significant reduction in the risk of dying from all causes and from cervical cancer was observed in those who received guideline treatment from 1999 onwards. In the stratified analysis by stage, the decreased all cause and cervical cancer mortality associated with receiving recommended concurrent chemo-radiotherapy was confined to patients with stage IIB-IVA disease (40\% and $36 \%$, respectively), which was consistent with the relevant clinical trials and the previous studies $[1-3,21,25-27]$. The contributing effects of other treatments (such as maintenance of haemoglobin levels), which was not assessable in the current dataset, cannot be excluded.

\section{Strengths}

This is the first population-based study of cervical cancer treatment in relation to the changes in the treatment guidelines both in Canada and internationally, which adjusted for potential confounders including tumour stage. The followup time in this study was longer than that previously reported in other studies with an overall median follow up time of 6.4 years [25].

\section{Limitations}

Similar to other population-based studies using administrative data $[14,25]$, this study has some limitations related to data availability. Therefore, we could only assess broad concordance with guidelines and could not take into account individual patient factors, specifically the effects of co-morbidities, poor performance status and patients' treatment preferences. Similarly, we could not assess other underlying factors related to practice that deviated from the guidelines, such as clinician referral practice, limitations in health care access and patient compliance. Accordingly, we are unsure to what extent each of these non-assessable factors contributed to the suboptimal treatment patterns observed. This is an area for future research if information on patients' comorbidity and performance status, such as the Eastern Cooperative Oncology Group (ECOG) score, is routinely recorded in the clinical charts. About $20 \%$ of cervical cancer patients diagnosed during the study period in Manitoba did not have their tumour stage recorded, and therefore were not included in the analysis. Missing stage information for these patients may have had an effect on the overall treatment patterns in accordance with the guidelines. Nevertheless, the extensive record linkage system in Manitoba allowed us to obtain demographic information for all patients as well as detailed information on treatment and stage for the majority (77 \%) of cervical cancer cases over a long period of time, and we were thereby able to examine the effect of guidelinerecommended treatment on survival after adjusting for confounding effects due to age and stage of disease.

\section{Conclusions}

Consistent evidence from clinical trials investigating the survival benefit of concurrent chemo-radiotherapy on cervical cancer led to substantial revision of treatment guidelines. We found this resulted in a rapid increase in the use of concurrent chemo-radiotherapy and an associated significantly increased survival in women diagnosed with invasive stage IIB-IVA cervical cancer, although the effect of other changes in clinical practice on increased survival cannot be ruled out.

\section{Abbreviations}

FIGO: International Federation of Gynecology and Obstetrics; MCR: Manitoba Cancer Registry; HR: Hazard ratio; Cl: Confidence interval; AJCC: American Joint Committee on Cancer.

\section{Competing interests}

K.C. is co-PI of an investigator-initiated cervical screening trial in Australia which is partly funded by Roche Molecular Systems and Ventana Inc., USA. K.C. receives salary support from the National Health and Medical Research Council Australia (CDF 1007994). EK and AD have received travel grants from Merck. EK has consulted for Merck and GlaxoSmithKline. Other authors have no competing interests to declare. YJK received funding from Cancer Council NSW for her postgraduate study and has received a travel grant from the Association of Canadian Studies in Australia and New Zealand. The funding sources had no involvement in study design, analysis, or interpretation of results, writing of the manuscript or the decision to submit for publication.

\section{Authors' contributions}

YJK, DLO'Connell and KC contributed to the conception and design of the study and interpreted the data. $A A D, R L$ and EVK provided the data and assisted in interpretation of the data. YJK performed the statistical analyses and drafted the 
manuscript. DEG advised on the statistical analysis and interpretation of the results. All authors provided comment on the manuscript and approved the final manuscript.

\section{Acknowledgements}

We thank Ms. Grace Musto (CancerCare Manitoba) for extracting data from CancerCare Manitoba databases; and Mr. Sam Egger (Cancer Council NSW) for advising on the statistical programming and analysis. YJK received funding from Cancer Council NSW for her postgraduate study and has received a travel grant from the Association of Canadian Studies in Australia and New Zealand. The funding sources had no involvement in study design, analysis, or interpretation of results, writing of the manuscript or the decision to submit for publication.

\section{Author details}

${ }^{1}$ Prince of Wales Clinical School, the University of New South Wales, Sydney, NSW, Australia. ${ }^{2}$ Cancer Research Division, Cancer Council NSW, 153 Dowling Street, Woolloomooloo, NSW, Australia. ${ }^{3}$ Division of Gynecologic Oncology, CancerCare Manitoba, Winnipeg, MB, Canada. ${ }^{4}$ Epidemiology and Cancer Registry, CancerCare Manitoba, Winnipeg, MB, Canada. ${ }^{5}$ Community Health Sciences, University of Manitoba, Winnipeg, MB, Canada.

Received: 20 May 2014 Accepted: 24 August 2015

Published online: 22 September 2015

\section{References}

1. Keys HM, Bundy BN, Stehman FB, Muderspach LI, Chafe WE, Suggs 3rd CL, et al. Cisplatin, radiation, and adjuvant hysterectomy compared with radiation and adjuvant hysterectomy for bulky stage IB cervical carcinoma. New Engl J Med. 1999;340(15):1154-61.

2. Morris M, Eifel PJ, Lu J, Grigsby PW, Levenback C, Stevens RE, et al. Pelvic radiation with concurrent chemotherapy compared with pelvic and para-aortic radiation for high-risk cervical cancer. New Engl J Med. 1999;340(15):1137-43.

3. Rose PG, Bundy BN, Watkins EB, Thigpen JT, Deppe G, Maiman MA, et al. Concurrent cisplatin-based radiotherapy and chemotherapy for locally advanced cervical cancer. New Engl J Med. 1999;340(15):1144-53.

4. National Cancer Institute. $\mathrm{PDQ}^{\circledR}$ Cervical Cancer Treatment. Bethesda: National Cancer Institute; 2010. Available from: http://www.cancer.gov/ cancertopics/pdq/treatment/cervical/HealthProfessional.

5. Greater Metropolitan Clinical Taskforce (GMCT). Best Clinical Practice: Gynaecological Cancer Guidelines. NSW: NSW Department of Health; 2009.

6. British Columbia Cancer Agency. Cancer management guidelines: cervical cancer. [2009]; Available from: http://www.bccancer.bc.ca/healthprofessionals/professional-resources/cancer-management-guidelines/ gynecology/uterine-cervix\#Chemotherapy.

7. National Comprehensive Cancer Network. Clinical Practice Guidelines in Oncology - v.1.2010 - Cervical Cancer. 2009. Available at URL: http://www. nccn.org/professionals/physician_gls/pdf/cervical.pdf.

8. Lukka H, Hirte H, Fyles A, Thomas G, Fung FK, Johnson M. Primary treatment for locally advanced cervical cancer: Concurrent platinumbased chemotherapy and radiation. Practice Guideline Report \#4-5. Ontario: CancerCare Ontario; 2004. [updated June 2004; cited 2009 Jan. 22nd]; Available from: http://www.cancercare.on.ca/common/pages/ UserFile.aspx?serverld=6\&path=/File\%20Database/CCO\%20Files/PEBC/ pebc4-5f.pdf.

9. Hirte H, Strychowsky J, Oliver T, Fung-Kee-Fung M, Elit L, Oza AM. Chemotherapy for recurrent, metastatic, or persistent cervical cancer: a systematic review. Int J Gynecol Cancer. 2007;17(6):1194-204.

10. British Columbia Cancer Agency. BCCA Protocol Summary for Treatment of High Risk Squamous Carcinoma, Adenocarcinoma, or Adenosquamous Carcinoma of the Cervix with Concurrent Cisplatin and Radiation. [updated 01/01/2011; cited 2011 Jan. 27th]; Available from: http://www.bccancer.bc.ca/chemotherapyprotocols-site/Documents/Gynecology/GOCXCRT_Protocol_1Jun2014.pdf.

11. Denny L, Hacker N, Gori J, Jones III H, Ng HN, Pecorelli S. Staging Classifications and Clinical Practice Guidelines for Gynaecologic Cancers. FIGO Committee on Gynecologic Oncology. Intc J Gynecol Obstetr. 2000;70:207-312

12. Benedet $\mathrm{J}$, Bender $\mathrm{H}$, Jones 3rd H, Ngan HY, Pecorelli S. FIGO staging classifications and clinical practice guidelines in the management of gynecologic cancers. FIGO Committee on Gynecologic Oncology. Int J Gynaecol Obstetr. 2000;70(2):209-62.

13. Barbera L, Paszat L, Thomas G, Covens A, Fyles A, Elit $L$, et al. The rapid uptake of concurrent chemotherapy for cervix cancer patients treated with curative radiation. Int J Radiat Oncol Biol Phys. 2006;64(5):1389-94.

14. Pearcey R, Miao Q, Kong W, Zhang-Salomons J, Mackillop WJ. Impact of adoption of chemoradiotherapy on the outcome of cervical cancer in Ontario: results of a population-based cohort study. J Clin Oncol. 2007;25(17):2383-8.

15. Epidemiology \& Cancer Registry. CancerCare Manitoba; Available from: http://www.cancercare.mb.ca/home/cancer_research/epidemiology_ and_cancer_registry/.

16. Canadian Institute for Health Information. Canadian classification of health interventions (CCI). Volume 3 - Tabular list. Ottawa, Ontario, Canada 2009 [cited June 1st, 2010]; Available from: https://www.cihi.ca/en/ cci_vol3_2009_en.pdf.

17. Anderson RN, Miniño MM, Hoyert DL, Rosenberg HM. Comparability of cause of death between ICD-9 and ICD-10: Preliminary Estimates. National Vital Statistics Report. 2001;49(2).

18. Landis J, Koch G. The measurement of observer agreement for categorical data. Biometrics. 1977;33:159-74.

19. National Institutes of Health Consensus Development Conference statement on cervical cancer. Gynecol Oncol. 1997;66(3):351-61.

20. Giordano SH, Duan Z, Kuo YF, Hortobagyi GN, Freeman J, Goodwin JS, et al. Impact of a scientific presentation on community treatment patterns for primary breast cancer. J Natl Cancer Inst. 2006;98(6):382-8.

21. Trimble EL, Harlan LC, Gius D, Stevens J, Schwartz SM. Patterns of care for women with cervical cancer in the United States. Cancer. 2008;113(4):743-9.

22. Green J, Kirwan J, Tierney J, Vale C, Symonds P, Fresco L, et al. Concomitant chemotherapy and radiation therapy for cancer of the uterine cervix. Cochrane Database of Systematic Reviews. 2005(3):Art. No.: CD002225. doi:10.1002/14651858.CD002225.pub2

23. Chow E, Hoskin P, Mitera G, Zeng L, Lutz S, Roos D, et al. Update of the international consensus on palliative radiotherapy endpoints for future clinical trials in bone metastases. Int J Radiat Oncol Biol Phys. 2012;82(5):1730-7.

24. Howell E, Chen YT, Moradi M, Concato J. Cervical cancer practice patterns and appropriateness of therapy. Am J Obstet Gynecol. 2000;183(2):407-13. Epub 2000/08/15

25. van der Aa MA, Siesling S, v d Poll-Franse LV, Schutter EM, Lybeert ML, Coebergh JW, et al. Age-specific differences in the treatment of cervical cancer in the east and the south of The Netherlands 1989-2004. Eur J Obstetr Gynecol Reprod Biol. 2009;147(1):78-82.

26. Whitney CW, Sause W, Bundy BN, Malfetano JH, Hannigan EV, Fowler Jr WC, et al. Randomized comparison of fluorouracil plus cisplatin versus hydroxyurea as an adjunct to radiation therapy in stage IIB-IVA carcinoma of the cervix with negative para-aortic lymph nodes: a Gynecologic Oncology Group and Southwest Oncology Group study. J Clin Oncol. 1999;17(5):1339-48

27. Peters 3rd WA, Liu PY, Barrett 2nd RJ, Stock RJ, Monk BJ, Berek JS, et al. Concurrent chemotherapy and pelvic radiation therapy compared with pelvic radiation therapy alone as adjuvant therapy after radical surgery in high-risk early-stage cancer of the cervix. J Clin Oncol. 2000;18(8):1606-13.

\section{Submit your next manuscript to BioMed Central and take full advantage of:}

- Convenient online submission

- Thorough peer review

- No space constraints or color figure charges

- Immediate publication on acceptance

- Inclusion in PubMed, CAS, Scopus and Google Scholar

- Research which is freely available for redistribution 